\title{
Datenkompetenz durch edukatives Privacy Nudging: Zentrale Prinzipien und Effekte auf Lernprozesse
}

Andreas Janson, Leonie Kreidel, Sofia Schöbel, Gerrit Hornung, Matthias Söllner und Marco Leimeister

\begin{abstract}
Die Digitalisierung verändert nicht nur unser privates, sondern auch unser Arbeitsleben. Immer mehr Daten über Individuen sind online verfügbar und werden für die Nutzung von bestimmten Online Services vorausgesetzt. Hierbei verlieren die Nutzenden oft den Überblick, wie und welche Daten sie von sich preisgeben. Dies birgt nicht nur das Risiko der Preisgabe von privaten Daten, sondern auch unternehmensseitig das Risiko, dass wichtige Daten außerhalb des Unternehmens veröffentlicht werden. Hier kann das Konzept des digitalen Nudgings angewandt werden, welches nunmehr auch als edukatives Privacy Nudging genutzt werden kann. Das Konzept bezweckt, Individuen durch bestimmte Elemente wie beispielsweise Defaults zu Verhaltensänderungen anzuregen. Dieser Beitrag hat zum Ziel, einen Überblick darüber zu geben, welche Privacy Nudges es im edukativen Bereich gibt und wie diese mit verschiedenen Lerntheorien in Einklang gebracht werden können. Der Text soll Forschern und Praktikern eine Orientierung geben, selbst edukative Privacy Nudges zu gestalten und endet mit einem Fallbeispiel, das aufzeigt wie einfache Gestaltungsänderungen Individuen dazu anregen können, sich privatheitsfreundlicher zu verhalten und sorgsamer mit ihren privaten Daten umzugehen.
\end{abstract}

\section{Einleitung und Motivation}

Die fortschreitende Digitalisierung und Entwicklungen von Informationstechnologien ermöglichen eine einfache und zunehmende weltweite Vernetzung. Dies hat Auswirkungen auf unsere Gesellschaft und Wirtschaft. Wertschöpfungsprozesse und Arbeitsweisen in Unternehmen verändern sich grundlegend. Zentral für diese Art der Wertschöpfung sind Daten, die 
als zentraler ökonomischer Motor agieren, beispielsweise um Angebote von sozialen Netzwerken und anderen Plattformen bestmöglich auf eine bestimmte Gruppe von Nutzenden anzupassen (Krasnova et al. 2010). Mit dieser Entwicklung gehen einerseits enorme Innovationspotenziale einher, beispielsweise die angesprochenen datengetriebenen Geschäftsmodelle. Andererseits entstehen auch große Risiken, welche die Preisgabe und den Umgang mit personenbezogenen Daten in der Plattformökonomie betreffen. Plattformen aller Art sind davon abhängig, dass möglichst viele oder zumindest genug relevante Daten von Nutzenden preisgegeben werden, wodurch beispielsweise das von Zuboff (2019) beschriebene Phänomen des Überwachungskapitalismus entstehen kann.

Entsprechend sind Konzepte notwendig, um mit dem Spannungsverhältnis zwischen Privatheit und den Innovationspotenzialen der Datenökonomie umgehen zu können (Acquisti et al. 2015). Der systematische Aufbau von Datenkompetenz (im Sinne einer spezifischen Form der Medienkompetenz) und die technische Unterstützung entsprechender Lernprozesse zur Sicherstellung eines reflektierten und souveränen Umgangs mit Daten können hierbei als zentrale Instrumente dienen, um diesem Spannungsverhältnis zu begegnen und Individuen ein für sie angemessenes Verhältnis von Privatheit und Datenpreisgabe zu ermöglichen. Gleichzeitig sind Umsetzungsinstrumente notwendig, um diese Kompetenzen zu vermitteln. Dabei gehen wir davon aus, dass klassische Bildungsträger diese nicht allein mit hergebrachten Methoden vermitteln können, sondern proaktive Prozesse wie edukative Nudging-Ansätze im Rahmen der Nutzung von digitalen Plattformen benötigt werden.

Die zentrale Grundannahme basiert auf dem Verständnis, dass Datenkompetenz eine wichtige Voraussetzung ist, damit Nutzende von digitalen Plattformen reflektierte Entscheidungen über die Preisgabe personenbezogener Daten treffen können. Beispielsweise wurde im Rahmen des sogenannten Privacy Paradox festgestellt, dass Menschen zwar abstrakt den Schutz ihrer Privatheit stark fokussieren, sie aber dennoch teilweise entgegengesetzt handeln (Smith et al. 2011). Die Gründe dafür sind vielfältig und können u.a. im fehlenden Verständnis für die Konsequenzen der Verletzung von Privatheit, in der Unkenntnis, wie man seine Privatheit schützen kann, und in der Komplexität der technischen Instrumente zur Wahrung der Privatheit liegen (Coventry et al. 2016). Datenkompetenz betrifft nicht nur die Vorstellung, welche Daten preisgegeben werden, sondern auch das Verständnis davon, was mit komplexen Verfahren, z.B. Machine Learning, mit personenbezogenen Daten möglich ist (Jones 2019) und welche Akteure (Nutzende, Datenempfänger und Dritte) hiervon profitieren können. So kann aus der Preisgabe personenbezogener Daten hoch- 
wertiges Wissen geschaffen werden. Bisher fehlen aber ein tiefgreifendes Verständnis und entsprechende Handlungskompetenz bei Nutzenden (und Unternehmen), um die mögliche Wertrealisierung einer Preisgabe von (personenbezogenen) Daten einzuschätzen (Günther et al. 2017). Gleichwohl kann aber Datenkompetenz als Grundlage für souveränes Agieren im digitalen Raum dienen.

Ansatzpunkte zur Förderung von Datenkompetenz im Rahmen der Nutzung digitaler Angebote kann das sogenannte Privacy Nudging bieten, welches das Verhalten in digitalen Umgebungen vorhersehbar dahingehend beeinflussen soll, dass privatheitsfreundliche Entscheidungen getroffen werden (Adjerid et al. 2019, Acquisti 2009). Hier können edukative Nudges (Heidbrink/Klonschinski 2018), nicht nur das Verhalten beeinflussen, sondern durch eine Reflexion auch Lernprozesse befördern. Ziel dieses Beitrags ist es daher, zentrale Prinzipien des Nudgings zu präsentieren und die datenschutzrechtliche Erforderlichkeit und Zulässigkeit im Anschluss an existierende Diskussionen (Sandfuchs 2015, Sandfuchs/Kapsner 2018) zu behandeln. Des Weiteren werden ausgewählte edukative Nudges vor dem Hintergrund zentraler Lerntheorien dargestellt und diskutiert, ob diese zum Aufbau von Datenkompetenz geeignet sind. Dies wird im Rahmen einer Fallstudie am Beispiel der mobilen Applikation Snapchat beleuchtet, welche aus Sicht des Datenschutzes und der jungen Gruppe von Nutzenden einschlägig ist, um das Thema der edukativen Privacy Nudges zu illustrieren.

\section{Theoretischer Hintergrund - Nudges, Digital Nudges und Privacy Nudges}

Der Ansatz des Nudgings kommt aus den Verhaltenswissenschaften und wurde federführend durch Richard Thaler und Cass R. Sunstein geprägt (Thaler/Sunstein 2008). Nudges versuchen durch externe Veränderungen des Handlungsrahmens Personen in Richtung bestimmter Handlungsoptionen zu „stupsen“ (englisch: to nudge), während die anderen Handlungsmöglichkeiten weiterhin offenstehen und somit die Entscheidungsfreiheit des Individuums erhalten bleibt. Ein Individuum wird lediglich zu einer Handlungsoption "genudged“, deren Folgen - aus Sicht des Nudgenden, d.h. wirklich oder vermeintlich - dem Interesse des Individuums am besten entsprechen. 


\subsection{Denkmodi im Nudging}

Nudges sind daher im Prinzip des libertären Paternalismus verankert (Mirsch et al. 2018). Es gibt viele unterschiedliche Szenarien, in denen Verhaltensänderungen mit Hilfe von Nudges herbeigeführt werden können. Sie können im Online-, sowie auch im Offline-Bereich genutzt werden und umfassen eine Vielzahl an Ansätzen. Im Offline-Bereich beeinflusst beispielsweise in einer Mensa der Ort, an dem ein Essen präsentiert wird, die Anzahl der Personen, die sich für dieses Essen entscheiden: Steht die vegetarische Option am Anfang oder wird die Aufmerksamkeit mit anderen Mitteln auf diese gelenkt, entscheiden sich mehr Menschen für diese Option. Nudges im Online-Bereich übertragen dieses Prinzip auf den digitalen Raum und lenken mit Hilfe von entsprechenden Designelementen in der Benutzeroberfläche die Individuen in die gewünschte Richtung (Weinmann et al. 2016), beispielsweise durch das Setzen von Voreinstellungen bei der erstmaligen Nutzung von Informationssystemen (Schöbel et al. 2020a).

Der Ansatz des libertären Paternalismus geht davon aus, dass Menschen nicht immer nach ihrem besten Interesse wählen und bezieht sich dabei auf die Theorie der zwei Systeme von Kahnemann und Tversky, welche zwei Denkmodi darstellen: die System 1- und die System 2-Denkweise (Kahneman 2011). Diese beiden unterschiedlichen Systeme beschreiben zwei mögliche Wege, die das Gehirn bei der kognitiven Verarbeitung von Reizen und Aufgaben einschlagen kann. Es sind zwei Systeme des Denkens. Die Denkweise von System 1 arbeitet automatisch und schnell, weitgehend mühelos und ohne willentliche Steuerung. Dagegen agiert die Denkweise von System 2 bewusst gesteuert, angestrengt und langsamer, womit das Denken von System 2 in der Lage ist, sich mit komplexen Problemen auseinanderzusetzen. Der Einsatz von System 2 erfordert die Aufmerksamkeit und die Konzentration von Individuen. Beides ist im Alltag schwierig und anstrengend aufrechtzuerhalten. System 1 macht demgegenüber Gebrauch von sogenannten Heuristiken, also verkürzten kognitiven Operationen, mit denen im Alltag ressourcensparend Schlussfolgerungen gezogen werden können, ohne dass komplizierte kognitive Vorgänge verlangt werden. Beide Systeme können, falls notwendig, gleichzeitig aktiv sein und zusammenarbeiten. Die Theorie geht davon aus, dass durch den Einsatz der Heuristiken vor allem in komplexen Situationen zwar rasche, zugleich aber voreilige und systematisch verzerrte Entscheidungen getroffen werden und Individuen per se anfällig für Urteilsfehler (Biases) sind (Kahneman 2011). Zur Verdeutlichung dieser Urteilsfehler wird als Beispiel der Ankereffekt dargestellt. Dieser Effekt tritt auf, wenn einer Schätz- 
frage vorrausgehend eine unbestimmte Zahl dargeboten wird. Beispielsweise gaben Englisch und Mussweiler (2001) 16 Richtern, die mindestens 15 Jahre im Amt waren, Material in welchem ein Delikt geschildert wurde. Anschließend bekamen diese die Information, ein Informatikstudent schlüge ein Strafmaß von 12 Monaten (Gruppe 1) oder 32 Monaten (Gruppe 2) vor. Die Richter sollten die Angemessenheit des Vorschlags beurteilen und anschließend selbst ein Strafmaß festsetzen. Die Richter in Gruppe 1 nannten durchschnittlich 28 Monate, die in Gruppe 235 Monate als angemessenes Strafmaß. Die genannten Monate blieben im Anschluss also näher bei der Anzahl an Monaten, die den Richtern im Voraus vorgeschlagen wurde (Enough/Mussweiler 2001). Dies bedeutet jedoch nicht, dass Individuen grundsätzlich irrational und unberechenbar sind, sondern dass man vielmehr mit einer systematischen, vorhersehbaren Abweichung vom rationalen Verhalten rechnen kann (Hertwig/Grüne-Yanoff 2017).

Nudges machen sich diese Urteilsfehler zunutze, indem sie die Auswahlmöglichkeiten so designen, dass das System 1 der Individuen die angestrebte Entscheidung auswählt. Manche Nudges erfordern auch die Mitarbeit des System 2. Bei diesen wird davon ausgegangen, dass sie einen langfristigen Lerneffekt hervorrufen (siehe auch zum Konzept des verwandten Boostings: Hertwig/Grüne-Yanoff 2017).

\subsection{Privacy Nudging}

Das Ziel der Privacy Nudges ist es, den Entscheidungsprozess der Individuen in Richtung „besserer“ Entscheidungen in Hinblick auf ihre Privatheit zu lenken und sie zu befähigen, ihre informationelle Selbstbestimmung zu berücksichtigen. Aufgrund von Biases werden beispielsweise personenbezogene Daten oftmals unüberlegt offengelegt, da das Risiko einer Verwendung der offengelegten Daten (Überwachung, Profilbildung etc.) mental weniger präsent ist (Verfügbarkeitsheuristik). Privacy Nudges setzen dabei an den beiden Denkmodi an, indem sie Heuristiken ausnutzen, beispielsweise durch privatheitsfreundliche Defaults, oder ihnen entgegenwirken, beispielsweise indem Denkprozesse bewusst nicht abgekürzt, sondern Reflexionsprozesse bei der Datenpreisgabe angestoßen werden (Weinmann et al. 2016).

Die Diskrepanz zwischen der hohen Bedeutung eigener Privatheit und dem dazu gegenläufigen Preisgeben von personenbezogenen Daten kann auf fehlende Handlungskompetenz und automatische, emotionale Entscheidungen zurückgeführt werden (Kühling/Martini 2016). Das Verhalten von Individuen bei der Preisgabe von Daten ist oftmals irrational. Pri- 
vacy Nudges können diese Irrationalität im Verhalten nutzen, um Privatheit in unterschiedlichen Szenarien zu fördern.

Privacy Nudging-Konzepte („Anstupser“) sollen das Verhalten von Nutzenden dahingehend beeinflussen, dass sie privatheitsfreundlichere Entscheidungen treffen. In diesem Sinne soll ein bewusster Umgang mit personenbezogenen Daten gefördert werden, um informationelle Selbstbestimmung sicherzustellen (Acquisti et al. 2017). Tabelle 1 stellt mögliche Elemente für den Einsatz der Privacy Nudges in digitalen Arbeitssystemen dar, welche durch Konzeptentwickler ausgewählt und gestaltet werden können (siehe hierzu und im Folgenden die Forschungsergebnisse von Schomberg et al. 2019, welche als Grundlage in diesem Beitrag dienen).

Tab.1: Beispiele digitaler Privacy Nudges im Arbeitsumfeld (Schomberg et al. 2019)

\begin{tabular}{|l|l|}
\hline Privacy Nudge & Beispiel \\
\hline Default & $\begin{array}{l}\text { Privat } \\
\text { Deine Channels werden standardmäbig als privat singestellt. } \\
\text { Geschlossene channels sind nur auf Einladung zugänglich und } \\
\text { erscheinen nicht in der Channel-Liste. }\end{array}$ \\
\hline Framing & $\begin{array}{l}\text { Privat } \\
\text { Geschlossene Channels sind nur auf Einladung zugänglich und } \\
\text { erscheinen nicht in der Channel-Liste. }\end{array}$ \\
\hline Information & $\begin{array}{l}\text { Im Durchschnitt können 38 } 38 \\
\text { Personen deine Nachrichten sehen. }\end{array}$ \\
\hline Feedback & $\begin{array}{l}\text { Du hast } 80 \% \text { deiner persönlichen Informationen } \\
\text { angegeben }\end{array}$ \\
\hline Zeitverzögerung & $\begin{array}{l}\text { Die Nachricht wird in } 5 \text { Sekunden gesendet } \\
\text { Bearbeiten }\end{array}$ \\
\hline Soziale Normerfen \\
\hline
\end{tabular}

\section{Default}

Als Default Nudge werden Standardeinstellungen im System beschrieben (Acquisti et al. 2017), welche meist als voreingestellte Optionen bevorzugt und nur selten verändert werden (Status-quo Bias). Individuen nutzen Defaults als Informationsquelle und zugleich als Referenzpunkt für das Abwägen von Entscheidungsoptionen. Defaults können tendenziell als die stärksten Nudges bewertet werden (Hummel/Maedche 2019) und gelten auch im Privacy Nudging als sehr effektiv, da Individuen in digitalen Um- 
gebungen die Privatheits-Einstellungen oftmals nicht nach ihren eigenen Bedürfnissen modifizieren. Defaults sind effektiv, weil sie nur einen sehr geringen kognitiven Beitrag und gar kein oder ein nur geringes Handeln des Individuums erfordern, zugleich aber dennoch die privaten Daten schützen.

\section{Framing}

Farbelemente können als Framing-Nudge verwendet werden, wobei farbliche Hinterlegungen die Aufmerksamkeit auf ausgewählte Elemente lenken, um bestimmte Entscheidungsalternativen hervorzuheben und so attraktiver erscheinen zu lassen (Turland et al. 2015). Die Schaltfläche zur Datenfreigabe kann zum Beispiel gegenüber den anderen Schaltflächen farblich hervorgehoben werden. Im hier gezeigten Beispiel wird der „privat"-Button in grüner Farbe markiert. Dies trägt dazu bei, dass Individuen bevorzugt diese Option wählen. Im Rahmen der digitalen Arbeit wären sensible Daten nun ausschließlich für eine bestimmte Zielgruppe oder nur für das Individuum selbst zugänglich (Almuhimedi et al. 2015). FramingNudges haben den großen Vorteil, dass diese ohne großen Aufwand in das Design integriert werden können und das Individuum schnell und effektiv dazu bewegen, wichtige Entscheidungen in Bezug auf seine Privatheit zu überdenken.

\section{Information}

Häufig ist die Wahrscheinlichkeit einer Verletzung der Privatheit nicht nachvollziehbar und wird deshalb oft unterschätzt. Dies kann auf die Repräsentationsheuristik zurückgeführt werden, welche die Gefahr beschreibt, dass Individuen die Häufigkeit der Beobachtungen eines Ereignisses fälschlicherweise mit dessen Eintrittswahrscheinlichkeit in Verbindung bringen. Dabei wird aus einem Prozess ein kleiner Ausschnitt als repräsentativ für den ganzen Prozess angesehen und schnell als Muster für alle Ereignisse anerkannt. Einen weiteren Einfluss stellt die Verfügbarkeitsheuristik dar, bei der Entscheidungen auf Informationen gestützt werden, die mental leicht verfügbar sind (Acquisti et al. 2017, Tversky/Kahneman 1974). Das Individuum über Risiken und Konsequenzen seines Handelns aufzuklären, ist deshalb eine effektive Methode, um zu verhindern, dass diese Heuristiken greifen. Diese Informationen kann das Individuum nutzen, um eine fundierte Entscheidung in Bezug auf die eigene Privatheit zu treffen. 


\section{Feedback}

Die Bereitstellung von Feedback als Privacy Nudge weist ein Individuum auf sein bisheriges Nutzungsverhalten hin. Dadurch entsteht ein Bewusstsein über die bisherigen Entscheidungen und ihre Konsequenzen. Ein Fortschritts-Balken, welcher z.B. beim Registrierungsprozess die Stärke eines Passworts illustriert oder die Menge der eingegebenen Daten im Profil widerspiegelt, ist ein Beispiel für einen Feedback-Nudge. Hier bewegen solche Nudges Individuen spielerisch dazu, ein komplexeres Passwort zu wählen oder weniger Daten im System zu hinterlegen (Khern-am-nuai et al. 2017). Das Design der Nudges, also die Art und Weise ihrer Darstellung, ist bei diesen Nudges ausschlaggebend für eine erfolgreiche Intervention. Besonders effektiv sind hier Textbenachrichtigungen ohne Ton, welche die User-Experience nicht einschränken (Micallef et al. 2017).

\section{Zeitverzögerung}

Das „Hyperbolic Discounting“ beschreibt die Tendenz eines Individuums, den unmittelbaren Nutzen einer Handlung zu überschätzen und später eintretende Kosten zu unterschätzen. Dies kann dazu führen, dass bei digitalen Entscheidungen über die persönliche Privatheit risikoreiche und wenig durchdachte Entscheidungen getroffen werden, ohne die späteren Konsequenzen der Handlung zu bedenken. Diesem Prozess kann mit Hilfe von Zeitverzögerungen als Privacy Nudges entgegengewirkt werden (Wang et al. 2014). Beispielsweise wird ein Countdown von fünf Sekunden eingesetzt, bevor eine Nachricht mit riskanten Inhalten im Firmennetzwerk veröffentlicht wird. Während dieser Zeit besteht die Möglichkeit das Senden der Nachricht abzubrechen und diese zu bearbeiten oder die Wartezeit zu beenden und die Nachricht direkt zu senden. Ziel des Nudges ist es, das Individuum dazu zu bewegen, weniger impulsiv zu handeln und den Inhalt der Nachricht sowie mögliche negative Konsequenzen zu überdenken. Die hohe Effektivität dieses Nudges sollte mit den potentiellen negativen Effekten wie z.B. einer erhöhten kognitiven Last abgewogen werden (Hu et al. 2017).

\section{Soziale Norm}

Individuen orientieren sich oftmals an dem Verhalten ihrer Mitmenschen, wenn sie Entscheidungen treffen müssen. Dies gilt auch für digitale Entscheidungen, welche die Privatheit der Individuen betreffen. Auf diesem Prinzip der sozialen Normen basiert die Wirkung von sogenannten sozialen Nudges (Coventry et al. 2016, Chang et al. 2016). Wenn ein Individu- 
um beispielsweise die Information erhält, dass $75 \%$ seiner Kollegen die private Telefonnummer nicht im Arbeitsprofil angegeben haben, zieht der individuelle Nutzende diese Information als Referenzpunkt für sein eigenes Handeln heran (Ankerheuristik). Die Wirksamkeit dieses Nudges kann laut einer Studie zu Social Nudges jedoch auch umgekehrt wirken und Individuen dazu veranlassen, mehr persönliche Daten preiszugeben. Falls die Mehrheit den Zugriff einer App auf bestimmte Daten zulässt, könnten Individuen dazu verleitet werden, sich ebenso zu verhalten (Zhang/Xu 2016). Es ist deshalb wichtig, diese Nudges mit Bedacht zu verwenden, um Individuen zu besseren Entscheidungen bezüglich des Schutzes ihrer Daten zu bewegen.

\subsection{Rechtliche Perspektive auf das Nudging}

Das Grundkonzept des Nudgings wurde in Auseinandersetzung mit imperativen Formen der (rechtlichen) Regulierung entwickelt; nicht umsonst ist einer der Väter der Idee, Cass Sunstein, Rechtswissenschaftler. Schon in seinen Untersuchungen, aber auch in der deutschen Diskussion (zuletzt z.B. Gerg 2019), wird als rechtliches und ethisches Grundproblem des Nudgings die Gefahr der Bevormundung, des Paternalismus, hervorgehoben. Ein solches Vorgehen kann prinzipiell gerechtfertigt werden, wenn es dem Schutz Dritter oder anerkannten Gemeinwohlinteressen (z.B. dem Umweltschutz) dient. Es wird aber problematisch, wenn es zu erzieherischen Zwecken eingesetzt wird. Sunstein begegnet entsprechenden Vorwürfen mit dem Konzept des „libertären“, also freiheitswahrenden Paternalismus und hebt hervor, die Entscheidungsfreiheit des Einzelnen werde im Ergebnis nicht behindert, sondern sogar befördert, weil er "gestupst“ werde, Entscheidungen zu fällen, die seinen Interessen dienen. Freilich wird das Paternalismus-Problem damit nur auf eine andere Ebene verschoben: Die Entscheidung, was im Interesse des einzelnen Betroffenen ist, wird von Dritten (politische Entscheider, Unternehmen, Programmierer oder andere Akteure) getroffen. Ob und gegebenenfalls unter welchen Voraussetzungen dieses Vorgehen rechtlich akzeptabel ist, ist weiterhin Gegenstand grundsätzlicher Kontroversen.

Bezogen auf das Datenschutzrecht und den Sonderfall der Privacy Nudges bedeutet dies: Gerade mit Blick auf die verfassungsrechtliche Fundierung im Recht auf informationelle Selbstbestimmung ist es prinzipiell problematisch, Nutzenden ein - dann nicht selbst-, sondern eben fremdbestimmtes - Maß an Datenschutz vorzugeben, also eine „Privatheit wider Willen“ (Sandfuchs 2015). Einen Ansatz zur Rechtfertigung bildet ein Be- 
gründungsstrang des Bundesverfassungsgerichts im Volkszählungsurteil von 1983, in dem es das Recht auf informationelle Selbstbestimmung (auch) als „elementare Funktionsbedingung eines auf Handlungsfähigkeit und Mitwirkungsfähigkeit seiner Bürger begründeten freiheitlichen demokratischen Gemeinwesens" bezeichnet hat. Aus dieser Gemeinwohlfundierung lässt sich zumindest ein grundsätzliches Argument für einen Privatheitsschutz auch ohne oder sogar gegen den Willen des Individuums ableiten, das aber noch weiterer Ausarbeitung bedarf. Ähnliches gilt für das Ziel des Schutzes Dritter, das etwa eingreifen könnte, wenn in bestimmten Situationen die reale Gefahr besteht, dass eine unreflektierte massenhafte Datenpreisgabe Dritte unter Druck setzt, ebenfalls auf ihre Privatheit zu verzichten.

Jenseits solcher übergeordneten Interessen und Schutzaufgaben ist es dem liberalen Rechtsstaat grundsätzlich verwehrt, seine Bürgerinnen und Bürger vor sich selbst zu schützen oder sogar zu erziehen (Hillgruber 1992, Schwabe 1998, Krönke 2016). Informationelle „Selbst“-Bestimmung impliziert zumindest grundsätzlich auch das Recht, sorglos mit seinen Daten umzugehen, solange die erwähnten Grenzen des Gemeinwohls und der Rechte Dritter nicht überschritten werden. Dies gilt allerdings nur mit einer wichtigen Ausnahme: Das Recht kennt in vielen Bereichen Instrumente zum Schutz von Minderjährigen und einwilligungsunfähigen Personen vor sich selbst, hier also derjenigen, die ihre Privatheit zwar selbstbestimmt preisgeben, bei denen es aber in dieser Frage aus rechtlicher Sicht an der Entscheidungskompetenz fehlt (Sandfuchs 2015).

Der letzte Punkt ist für die DSGVO von besonderer Bedeutung, weil diese an vielen Stellen explizit die besondere Vulnerabilität von Minderjährigen und den daraus resultierenden hohen Schutzbedarf hervorhebt (Art. 6 Abs. 1 UAbs. 1 lit.f, Art. 8, Art. 12 Abs. 1, Art. 17 Abs. 1 lit.f, Erwägungsgründe 38, 58, 65, 71 und 75). Insofern eröffnet sich im Bereich von Online-Angeboten, die sich spezifisch an Minderjährige richten (sowie bei der schulischen Bildung) ein nicht nur sinnvolles, sondern auch rechtlich abgesichertes Einsatzfeld für Privacy Nudges.

Mit Blick auf die erwähnten Schutzziele wird man auch im Übrigen davon ausgehen müssen, dass Privacy Nudges, die keine übermäßige Willenseinschränkung beinhalten (bei denen der Betroffene also zwar gegebenenfalls mit etwas Aufwand, aber grundsätzlich problemlos alternative Handlungsoptionen wählen kann), nicht in unzulässiger Weise in die Autonomie der Grundrechtsträger eingreifen. Wenn also die DSGVO in den Art. $12 \mathrm{ff}$. die Informationspflichten der Verantwortlichen erheblich ausgeweitet und damit - wenn man so will - Informations-Nudges vorgibt, so ist dies unproblematisch, solange der Nutzende nicht gezwungen wird, 
sich im Detail und ungebührlich lange mit den Informationen zu befassen. Es dient sogar umgekehrt dem anerkennenswerten Ziel, eine uninformierte, und damit nicht selbstbestimmte Preisgabe der Privatheit zu verhindern, und damit dem Schutz der Selbstbestimmung (Sandfuchs 2015).

Es ist aus demselben Grund nicht grundrechtlich problematisch, wenn der europäische Gesetzgeber in Art. 25 Abs. 2 DSGVO eine Pflicht zu privatheitsfreundlichen Voreinstellungen vorgibt und damit verbindlich die Verwendung von Default Nudges anordnet (Schomberg et al. 2019). Dies gilt zumindest, solange es dem Nutzenden nicht durch die Gestaltung der Oberfläche unverhältnismäßig erschwert wird, die Voreinstellungen nach seinen eigenen Präferenzen zu verändern.

Im Ergebnis ist der Einsatz von Privacy Nudges damit im Falle von Art. 25 Abs. 2 DSGVO verpflichtend und in vielen anderen Szenarien der technischen Gestaltung von IT-Systemen ein sinnvolles Instrument zum Schutz der Privatheit der Nutzenden, wenn es in geeigneter Weise ihre Selbstbestimmung fördert und z.B. das Problem des Privacy Paradoxes adressiert (Sandfuchs/Kapsner 2018). Nudges können auch ein Instrument sein, um die Datenschutzgrundsätze des Art. 5 DSGVO umzusetzen und im Sinne der Rechenschaftspflicht (Art. 5 Abs. 2 DSGVO) diese Umsetzung nachzuweisen. Es handelt sich also - je nach Ausgestaltung - um technische und/oder organisatorische Maßnahmen, die der Verantwortliche nach dem Grundsatz des Datenschutzes durch Technikgestaltung (Art. 25 Abs. 1 DSGVO) ergreifen muss. Hierbei besteht zwar keine Pflicht zum Einsatz von Nudges, weil es insoweit einen Einschätzungs- und Auswahlspielraum des Verantwortlichen gibt. Edukative Nudges könnten in Zukunft hierbei aber eine relevante Rolle spielen.

\section{Lernen durch edukatives Nudging}

Eine lernförderliche Gestaltung von Privacy Nudges kann einen Lernprozess und das Verhalten von Lernenden kurz-, mittel- und langfristig anregen und Datenkompetenz aufbauen. Um eine langanhaltende Verhaltensänderung in Richtung auf Privatheit förderndes Verhalten zu erzielen, ist es notwendig, Lernende zum Lernen anzuregen und sie in ihrem Lernprozess aktiv zu unterstützen. Im Folgenden werden verschiedene Theorien dafür diskutiert, welche Prozesse für langanhaltendes Lernen verantwortlich sind. Die bedeutendsten werden kurz vorgestellt, in Beziehung zu den Privacy-Nudges gestellt und hinterfragt, ob nicht auch Nudges in der Lage sind, langanhaltende Verhaltensänderungen herbei zu führen. 


\subsection{Behavioristische Lerntheorie}

Die behavioristische Lerntheorie geht davon aus, dass ein Individuum durch einen Reiz oder eine Stimulation zu einer Reaktion veranlasst wird. Durch externes positives oder negatives Feedback wird diese Reaktion entweder verstärkt oder aber abgeschwächt und die Wahrscheinlichkeit derselben Reaktion bei erneuter Präsentation des Reizes oder Stimulus steigt oder sinkt (Skinner 1954). In Bezug auf Nudges kann hier bei dem externen Feedback angesetzt werden und beispielsweise durch Smileys ein positives oder negatives Feedback die Reaktion verstärken oder abschwächen. Dementsprechend müsste der Nudge wiederholt eingesetzt werden, um einen langfristigen Lerneffekt hervorzurufen. Auf Basis der behavioristischen Lerntheorie kann argumentiert werden, dass auch Nudges durch Wiederholung zu einem Lerneffekt und anhaltender Verhaltensänderung beitragen. Gleichzeitig sollte aber Erwähnung finden, dass insbesondere behavioristisches Lernen dem libertären Gedanken und der eigenen Entscheidungsfreiheit entgegensteht.

\subsection{Kognitivistische Lerntheorie}

Anders als die behavioristische Lerntheorie richtet die kognitivistische Lerntheorie den Fokus weniger auf das sichtbare Verhalten, sondern auf kognitive Informationsverarbeitungsprozesse. Diese Theorie postuliert, dass ein Individuum Informationen aktiv aufnimmt, diese verarbeitet, zu innerlich repräsentierten Wissenseinheiten abspeichert und dann an bereits vorhandenes Wissen anknüpft (Rösler 1983). Bei direkter Rückmeldung dienen die dabei übermittelten Informationen weniger als Verstärker, sondern eher als Informationsquelle für das Individuum (Krapp/ Weidenmann 2001). Als Nudge könnten dem Individuum genauere Informationen über die Bedeutung von Privatheit mitgeteilt werden, sodass das Individuum die Informationen auf den Umgang mit seinen eigenen Daten beziehen kann. Durch die kognitive Verarbeitung der Nudge-Informationen sind Informationen im Langzeitgedächtnis gespeichert. Eine Wiederholung der Informationen würde den Lerneffekt dennoch verstärken und festigen. Diese Art der Nudges könnte man den edukativen Nudges zuordnen, da hier eine Veränderung der Kognitionen oder des Wissenstandes zu einer Verhaltensänderung führt. 


\subsection{Sozial-kognitive Lerntheorie}

Die sozial-kognitive Lerntheorie Albert Banduras, auch Modelllernen genannt, kann in vier Phasen unterteilt werden und beginnt mit der Aufmerksamkeitsphase, in welcher das Individuum seine Aufmerksamkeit auf ein Modell lenkt und dessen Handlungen beobachtet (Janson et al. 2017). In der zweiten Phase speichert das Individuum die zuvor beobachteten Handlungen im Gedächtnis ab und ahmt diese in der dritten Phase nach, indem die Handlungen aus dem Gedächtnis reproduziert werden. In der vierten Verstärkungs- und Motivationsphase werden die nachgeahmten Handlungen des Individuums verstärkt, sobald ein Erfolg wahrgenommen wird (Bandura 1977). Nudges, welche Privatheit förderndes Verhalten modellieren und entsprechende soziale Normen aufnehmen, eignen sich im Rahmen dieser Lerntheorie besonders. Hier ist beispielsweise das Vorleben (Gupta/Bostrom 2013) von privatheitsfreundlichen Einstellungen durch Freunde und andere soziale Bezugsgruppen zu nennen, wonach sich Individuen richten können. Auch hier ist eine Wiederholung zur besseren Einprägung förderlich und die wiederholte Durchführung des Verhaltens kann dazu beitragen, dass das gewünschte Verhalten eine Gewohnheit für das Individuum wird (Bandura 1997). System 1 (s. 2.1 Denkmodi im Nudging) übernimmt die gewünschte Verhaltensoption und speichert diese als Standardverhalten ab.

\subsection{Konstruktivistische Lerntheorie}

Der konstruktivistische Ansatz definiert Lernen als individuellen und aktiven Prozess des Individuums. Der Fokus liegt auf individuellen, konstruktiven Prozessen in sozialen Interaktionen. Das bedeutet, dass das Wissen nicht von einer Person auf eine andere übertragen werden kann, sondern von jedem Individuum neu konstruiert wird. Anhand von Informationen schafft jedes Individuum sich eine subjektive Realität, welche stark von der individuellen Prägung abhängig ist. Die Informationen erhalten Bedeutung durch den Bezug zu relevanten Kontexten (Janson et al. 2019). Wichtig sind hierbei die intrinsische Motivation des Individuums, die Inhalte lernen zu wollen, das Vorwissen (Thiel de Gafenco et al. 2018), sein kultureller Hintergrund (Ernst et al. 2018) und seine Erfahrungen. Nudges, welche kognitive Prozesse anstoßen, sind in Bezug auf diese Theorie von Bedeutung. Ähnlich wie bei dem kognitivistischen Ansatz ist auch hier der Nudge repräsentativ im Gedächtnis gespeichert und eine Wiederholung würde den bestehenden Lerneffekt festigen und verstärken. Nudges, welche in diesem Kontext von Bedeutung sind, sind ebenfalls die edukativen Nudges. 


\section{Edukative Privacy Nudges im digitalen Umfeld}

Im Folgenden soll nun ergänzend dargestellt werden, wie die oben vorgestellten Lernprozesse in Zusammenhang mit den Privacy Nudges zu langanhaltenden Verhaltensänderungen führen können. Zur Übersicht wurden die Nudges nach den zugrundeliegenden Lerntheorien geordnet.

\section{Default und Framing}

Die Default- und Framing-Nudges können durch behavioristische Prozesse anhaltende Verhaltensänderung herbeiführen. Der Reiz, auf den eine Reaktion des Individuums folgt, wird im Vorhinein so modifiziert, dass bei diesen Nudges bereits die gewünschte Reaktion durch den Reiz angestoßen wird. Bei Default-Nudges ist dies die Standardeinstellung, welche oftmals ohne Änderung angenommen wird und jedenfalls dann als Reiz wirkt, wenn sie zuvor wahrgenommen (also nicht nur „weggeklickt“) wird. Die Framing-Nudges lenken mit Hilfe von Farbelementen die Aufmerksamkeit des Individuums auf die gewünschte Reaktion (Schomberg et al. 2019). Durch die daraus entstehenden privatheitsfreundlichen Auswirkungen seines Handelns erfährt das Individuum ein positives Feedback seines Verhaltens, und dieses wird verstärkt. Die Wahrscheinlichkeit, dass das Individuum das gewünschte Verhalten in Zukunft erneut ausführt, steigt (Skinner 1954).

\section{Information und Feedback}

Informations- und Feedback-Nudges können als unterstützende Elemente im Lernprozess für den Behaviorismus und den Kognitivismus eingeordnet werden. Das Individuum erhält eine direkte Reaktion auf seine Handlung, welche in Form von positivem Feedback verstärkend oder in Form von negativem Feedback abschwächend sein kann (Skinner 1954). So könnte ein Informationssystem beispielsweise am Ende jedes Tages ein klares Feedback darüber bereitstellen, wie viele Daten das Individuum geteilt hat und was dies bedeutet (Almuhimedi et al. 2015). Aus kognitivistischer Perspektive besitzen beide Ansätze ebenfalls das Potenzial, eine anhaltende Verhaltensänderung herbeizuführen. Das Individuum hat die Möglichkeit, die durch das Feedback erhaltenen Informationen zu verarbeiten, in bestehende Wissensstrukturen einzuordnen und abzuspeichern. Es ist so in der Lage, das erworbene Wissen zu abstrahieren und auf andere Kontexte anzuwenden (Krapp/Weidenmann 2001). 


\section{Soziale Norm}

Zu den Nudges, welche sich das Konzept der sozialen Norm zu Nutze machen, gehören jene, die dem Individuum soziale Normen spiegeln und solche, die das gewünschte Verhalten modellieren. Beide Arten gehören sowohl zum Kognitivismus als auch zum sozial-kognitiven Lernen. Wird das gewünschte Verhalten modelliert, hat das Individuum die Chance, das beobachtete privatheitsfreundliche Verhalten des Modells zu übernehmen und so eigene negative Erfahrungen zu umgehen (Bandura 1977). Die Informationen über die Auswirkungen des Verhaltens des Modells kann es so abspeichern und in Zukunft auf sein eigenes Verhalten übertragen. Werden soziale Normen zum Gegenstand des Nudgings, kann das Individuum das privatheitsfreundliche Verhalten, welches für einen Großteil der Personen leitend ist, als Referenzpunkt für seine eigenen Handlungen nutzen und hat so ebenfalls ein Modell zur Verfügung.

\section{Tabelle 2: Nudges und Lerntheorien}

\begin{tabular}{|l|l|l|}
\hline Nudge & Lerntheorie & $\begin{array}{l}\text { Mögliche Effekte für privatheits- } \\
\text { freundliches Verhalten }\end{array}$ \\
\hline Default & Behavioristische Lerntheorie & Keine Änderungen des Default Settings \\
\hline $\begin{array}{l}\text { Framing } \\
\text { Präsentation) }\end{array}$ & Behavioristische Lerntheorie & $\begin{array}{l}\text { Die Auswahl nehmen, die farblich als } \\
\text { privatheitsfreundlich hervorgehoben ist }\end{array}$ \\
\hline Feedback & $\begin{array}{l}\text { Behavioristische Lerntheorie, } \\
\text { kognitivistische Lerntheorie }\end{array}$ & $\begin{array}{l}\text { Rückmeldung über das Verhalten so lan- } \\
\text { ge bis das System meldet, dass privatheits- } \\
\text { freundliche Einstellungen vorliegen }\end{array}$ \\
\hline $\begin{array}{l}\text { Information } \\
\text { Soziale Einflüsse } \\
\text { kehavioristische Lerntheorie, } \\
\text { kognitivistische Lerntheorie } \\
\text { sozial-kognitive Lerntheorie } \\
\text { quenzen aufzeigt }\end{array}$ & $\begin{array}{l}\text { Die Option wählen, die entsprechend } \\
\text { quistive privatheitsfreundliche Konse- } \\
\text { genutzt hat }\end{array}$ \\
\hline $\begin{array}{l}\text { Nudging, das } \\
\text { kognitive Prozesse } \\
\text { anregt }\end{array}$ & Konstruktivistische Ansatz & $\begin{array}{l}\text { Langzeiteffekte, die mit Emotionen, } \\
\text { Erfahrungen und der Entwicklung einer } \\
\text { Meinung verbunden sind }\end{array}$ \\
\hline
\end{tabular}

\section{Nudges, die kognitive Prozesse auslösen}

Wenn Nudges in Form von Hinweisen verwendet werden, lösen diese kognitive Prozesse aus und können der konstruktivistischen Lerntheorie zu geordnet werden (siehe Tabelle 2 für den Vergleich der Theorien). Derartige Nudges induzieren Emotionen und Erfahrungen und generieren Bedeutung. Es wird davon ausgegangen, dass hierbei stabile Langzeiteffekte nachweisbar sind (Krapp/Weidenmann 2001). 
Aufbauend auf der Beschreibung der einzelnen Nudges und der Lerntheorien, bietet die nachfolgende Tabelle einen Einblick in die Zusammenhänge zwischen den verschiedenen Nudges, den Lerntheorien und möglichen Verhaltenseffekten.

Um den Einsatz der Nudges besser verstehen zu können, dient das nachfolgende Beispiel als Grundlage.

\section{Ansatzmöglichkeiten zu edukativen Nudges am Beispiel von Snapchat}

Snapchat ist ein sogenannter Instant-Messaging Dienst, der auf mobilen Endgeräten genutzt werden kann und es Nutzenden ermöglicht, Fotos und andere Medien für eine bestimmte Anzahl von Sekunden an andere Nutzende zu senden, bis die gesendeten Medien verschwinden.

Innerhalb von Snapchat gibt es zahlreiche Ansätze des Einsatzes von Nudges, mit denen Nutzende auf eine edukative Weise angeregt werden, ihr Verhalten zu verändern. Gleichzeitig zeigt die aktuelle Applikation aber, dass von diesen Möglichkeiten kaum Gebrauch macht wird. Abbildung 1 zeigt zwar Informationen zur Nutzung an, nudged aber zum bewussten Freigeben der Kontakte durch ein Framing, wobei der „Ok“-Button im Gegensatz zu dem „Nicht Erlauben“-Button hervorgehoben wird (bedingt durch das Betriebssystem Apple IOS).

\section{Abbildung 1. Informationsnudge}

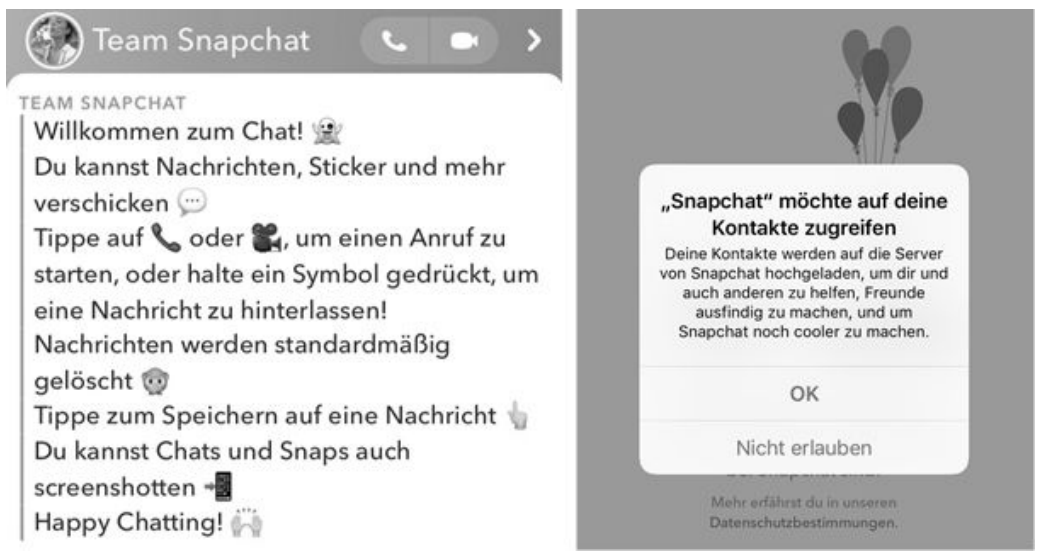

Quelle: Eigene Screenshots aus Snapchat App, Snap, Inc., https://apps.apple.com/de/app/snapchat/id447188370 
Innerhalb der Einstellungen verwendet Snapchat zwei weitere Nudges zum Standort teilen (siehe Abbildung 2). So wird bewusst die Entscheidung der Standortnutzung zum „Erlauben“ angestupst, gleichzeitig wird aber auch ein privatheitsfreundlicher Default implementiert, indem das Teilen des öffentlichen Standorts durch den sogenannten "Geistmodus“ abgeschaltet wird. Beiden Nudges gemein ist, dass die edukativen Aspekte im Hintergrund sind.

\section{Abbildung 2: Information und Default Nudge}
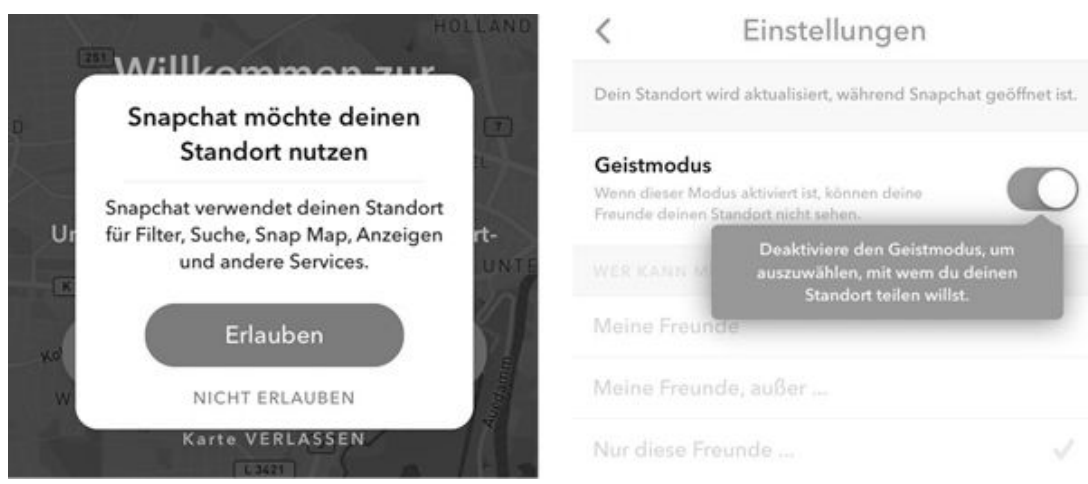

Quelle: Eigene Screenshots aus Snapchat App, Snap, Inc., https://apps.apple.com/de/app/snapchat/id447188370

Bis auf die Default Option sind die Einstellungen und der Umgang mit den Daten von Snap Chat Nutzenden durchaus als fragwürdig zu beurteilen, was den Schutz von Nutzerdaten angeht. Durch einfache Änderungen und Integration von Nudges können Nutzende jedoch edukativ so geschult werden, dass diese sensibler mit ihren Daten umgehen. Mögliche Beispiele, wie Snapchat seine Benutzeroberfläche privatheitsfreundlicher gestalten könnte, werden in Abbildung 3 vorgestellt. 
Abbildung 3: Mögliche privatheitsfreundliche Anpassungen von Snapchat (eigenes Beispiel für mögliche Erweiterungen)

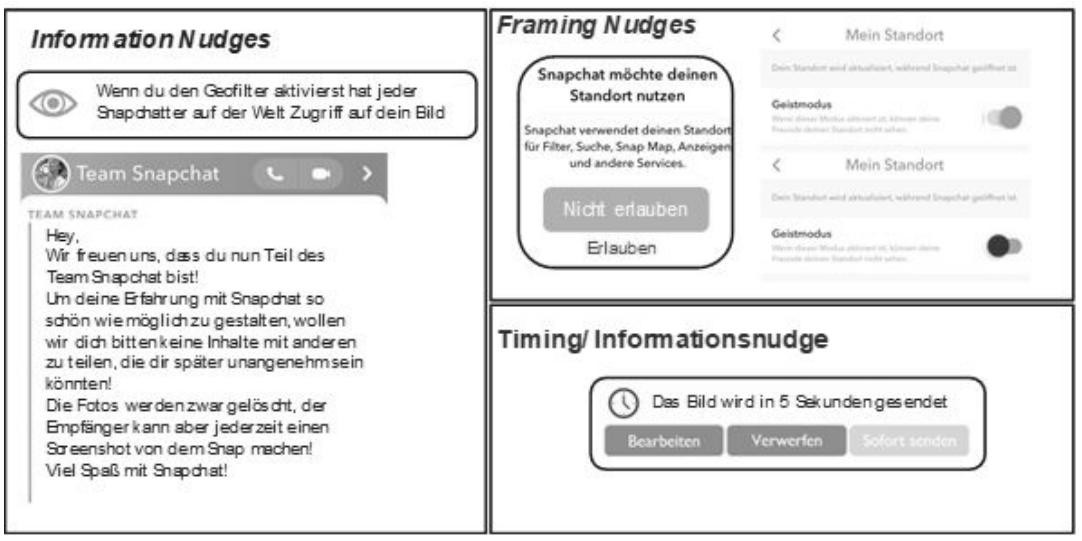

Basierend auf: Eigene Screenshots ais Snapchat App, Snap, Inc., https://apps.apple.com/de/app/snapchat/id447188370

Wie bereits zuvor vorgestellt, wäre die einfachste Option der Einsatz eines Informationsnudges in Kombination mit Framing. Anstelle der aus Sicht der Privatheit bedenklichen Option, sollte dann die privatheitsfreundliche hervorgehoben werden. Durch das Framing kann auch die sogenannte Geisterfunktion in Bezug auf die Privatheit verbessert werden, also die Nutzung des Systems ohne permanentes Teilen des eigenen Standorts. Hier kann die rote Farbe ein nicht privatheitsfreundliches Verhalten indizieren, die grüne hingegen ein privatheitsfreundliches Verhalten. Um das Grundprinzip des Versendens von Bildern besser zu steuern, könnte eine Zeitverzögerung an einen Informationsnudge gekoppelt werden, sodass dem Nutzenden noch einmal bewusst gemacht wird, was für ein Bild er dort teilt.

\section{Zusammenfassung und Würdigung}

Edukatives Privacy Nudging gewinnt immer mehr an Bedeutung. Besonders die vermehrte Preisgabe von Daten in Online Umgebungen erfordert, Individuen für einen anhaltenden achtsamen Umgang mit privaten Daten zu sensibilisieren. Hier kann das Konzept des digital Nudgings angewandt werden, welches als edukatives Nudging einen klaren Bezug zum Lernen herstellen kann. Nudges wie Defaults basieren beispielsweise auf der beha- 
vioristischen Lerntheorie und können durch ihren Einsatz Individuen dazu anregen, vorsorglicher zu agieren. Soziale Einflüsse hingegen können durch die Demonstration von Handlungsweisen anderer Individuen, basierend auf der sozial kognitiven und der kognitivistischen Lerntheorie, dazu beitragen, dass Individuen durch das Verhalten anderer Nutzender lernen, sich selbst privatheitsfreundlicher zu verhalten. Dieser Beitrag zeigte daher verschiedene edukative Privacy Nudges auf und bietet somit einen Ansatzpunkt für Forscher und Praktiker edukative Privacy Nudges zu gestalten. Limitiert ist dieser Beitrag durch die fehlende empirische Demonstration der Effektivität von edukativen Privacy Nudges, was jedoch einen Raum für künftige Forschungsstudien bieten kann, beispielsweise hinsichtlich der zielgruppenspezifischen Gestaltung durch spielerische Nudges (Schöbel et al. 2020b).

\section{Danksagung}

Dieser Beitrag wurde im Rahmen des Projekts „Nudger“ (www. nudger.de; Förderkennzeichen: 16KIS0890K; 16KIS0891) unter der Projektträgerschaft des VDI/VDE-IT erarbeitet und mit den Mitteln des Bundesministeriums für Bildung und Forschung gefördert. Zudem danken wir Melanie Schwede für die initiale Mitarbeit an der Beitragsidee. Die Verantwortung für den Inhalt dieser Veröffentlichung liegt bei den Autoren.

\section{Literatur}

Acquisti, Alessandro (2009): Nudging privacy: The behavioral economics of personal information. In: IEEE security \& privacy 7 (6), S. 82-85.

Acquisti, Alessandro / Brandimarte, Laura / Loewenstein, George (2015): Privacy and human behavior in the age of information. In: Science (New York, N.Y.) 347 (6221), S. 509-514. doi:10.1126/science.aaa1465.

Acquisti, Alessandro / Adjerid, Idris / Balebako, Rebecca / Brandimarte, Laura / Cranor, Lorrie F. / Komanduri, Saranga / Leon, Pedro G. / Sadeh, Norman / Schaub, Florian / Sleeper, Manya / Wang, Yang / Wilson, Shomir (2017): Nudges for privacy and security. In: ACM Comput. Surv. 50 (3), S.1-41. doi:10.1145/3054926.

Adjerid, Idris / Acquisti, Alessandro / Loewenstein, George (2019): Choice architecture, framing, and cascaded privacy choices. In: Management Science 65 (5), S. 2267-2290. doi:10.1287/mnsc.2018.3028. 
Almuhimedi, Hazim / Schaub, Florian / Sadeh, Norman / Adjerid, Idris / Acquisti, Alessandro / Gluck, Joshua / Cranor, Lorrie F. / Agarwal, Yuvraj (2015): Your location has been shared 5,398 times!: A field study on mobile app privacy nudging. In: Proceedings of the 33rd annual ACM conference on human factors in computing systems, S. 787-796.

Bandura, Albert (1977): Social learning theory. Englewood Cliffs: Prentice-Hall.

Bandura, Albert (1997): Self-efficacy. The exercise of control. New York: W.H. Freeman.

Chang, Daphne / Krupka, Erin L. / Adar, Eytan / Acquisti, Alessandro (2016): Engineering information disclosure. Norm shaping designs. In: Proceedings of the 2016 CHI Conference on Human Factors in Computing Systems. New York, NY, USA: Association for Computing Machinery (CHI '16), S. 587-597. doi:10.1145/2858036.2858346.

Coventry, Lynne. M. / Jeske, Debora / Blythe, John M. / Turland, James / Briggs, Pam (2016): Personality and social framing in privacy decision-making: A study on cookie acceptance. In: Frontiers in Psychology 7, S. 1-12.

Enough, Birte / Mussweiler, Thomas (2001): Sentencing under uncertainty. Anchoring effects in the courtroom. In: J Appl Soc Psychol 31 (7), S. 1535-1551. doi:10.1111/ j.1559-1816.2001.tb02687.x.

Ernst, Sissy-Josefina / Janson, Andreas / Söllner, Matthias / Leimeister, Jan Marco (2018): Mobiles Lernen in praktischen Trainings. Lernzielerreichung vor dem Hintergrund von Motivation und Akzeptanz der Zielgruppe. In: de Witt, Claudia / Gloerfeld, Christina (Hg.): Handbuch Mobile Learning. Wiesbaden: Springer VS, S. 409-431.

Gerg, Stephan (2019): Nudging. Verfassungsrechtliche Maßstäbe für das hoheitliche Einwirken auf die innere Autonomie des Bürgers. Tübingen: Mohr Siebeck (5).

Günther, Wendy Arianne / Rezazade Mehrizi, Mohammad H./ Huysman, Marleen / Feldberg, Frans (2017): Debating big data. A literature review on realizing value from big data. In: The Journal of Strategic Information Systems 26 (3), S. 191-209. doi:10.1016/j.jsis.2017.07.003.

Gupta, Saurabh / Bostrom, Robert (2013): An investigation of the appropriation of technology-mediated training methods incorporating enactive and collaborative learning. In: Information Systems Research 24 (2), S. 454-469. doi:10.1287/ isre.1120.0433.

Heidbrink, Ludger / Klonschinski, Andrea (2018): Nudges, Transparenz und Autonomie - Eine normativ gehaltvolle Kategorisierung von Maßnahmen des Nudgings. In: Vierteljahrshefte zur Wirtschaftsforschung 87 (1), S.15-27. doi:10.3790/ vjh.87.1.15.

Hertwig, Ralph / Grüne-Yanoff, Till (2017): Nudging and boosting. Steering or empowering good decisions. In: Perspectives on Psychological Science: A Journal of the Association for Psychological Science 12 (6), S.973-986. doi:10.1177/1745691617702496.

Hillgruber, Christian (1992): Der Schutz des Menschen vor sich selbst. München: Vahlen. 
$\mathrm{Hu}$, Paul Jen-Hwa / Hu, Han-fen / Fang, Xiao (2017): Examining the mediating roles of cognitive load and performance outcomes in user satisfaction with a website: A field quasi-experiment. In: MIS Quarterly 41 (3), S. 975-987.

Hummel, Dennis / Maedche, Alexander (2019): How effective is nudging? A quantitative review on the effect sizes and limits of empirical nudging studies. In: Journal of Behavioral and Experimental Economics 80, S. 47-58. doi:10.1016/ j.socec.2019.03.005.

Janson, Andreas / Söllner, Matthias / Leimeister, Jan Marco (2017): Individual appropriation of learning management systems - Antecedents and consequences. In: AIS Transactions on Human-Computer Interaction 9 (3), S. 173-201.

Janson, Andreas / Söllner, Matthias / Leimeister, Jan Marco (2020): Ladders for learning. Is scaffolding the key to teaching problem solving in technology-mediated learning contexts? In: Academy of Management Learning \& Education 19 (4), S. 439-468. doi:10.5465/amle.2018.0078.

Jones, Matthew (2019): What we talk about when we talk about (big) data. In: The Journal of Strategic Information Systems 28 (1), S.3-16. doi:10.1016/ j.jsis.2018.10.005.

Kahneman, Daniel (2011): Thinking, fast and slow. New York: Farrar, Straus and Giroux.

Khern-am-nuai, Warut / Yang, Weining / Li, Ninghui (2017): Using context-based password strength meter to nudge users' password generating behavior: A randomized experiment. In: Hawaii International Conference on System Sciences 2017 (HICSS-50). Online verfügbar unter: https:/www.researchgate.net/publication/31712 0932_Using_Context-Based_Password_Strength_Meter_to_Nudge_Users\%27_P assword_Generating_Behavior_A_Randomized_Experiment (Abfrage am: 6.10.2020).

Krapp, Andreas / Weidenmann, Bernd (Hg.) (2001): Pädagogische Psychologie: Weinheim: Beltz.

Krasnova, Hanna / Spiekermann, Sarah / Koroleva, Ksenia / Hildebrand, Thomas (2010): Online social networks. Why we disclose. In: Journal of Information Technology 25 (2), S. 109-125. doi:10.1057/jit.2010.6.

Krönke, Christoph (2016): Datenpaternalismus. Staatliche Interventionen im OnlineDatenverkehr zwischen Privaten, dargestellt am Beispiel der Datenschutz-Grundverordnung. In: Der Staat 55 (3), S. 319-351.

Kühling, Jürgen / Martini, Mario (2016): Die Datenschutz-Grundverordnung. Revolution oder Evolution im europäischen und deutschen Datenschutzrecht? In: Europäische Zeitschrift für Wirtschaftsrecht (EuZW), S. 448-453.

Micallef, Nicholas / Just, Mike / Baillie, Lynne / Alharby, Maher (2017): Stop annoying me! In: Alessandro Soro (Hg.): OzCHI 2017 - Human Nature. Proceedings of the 29th Australian Computer-Human Interaction Conference (OzCHI 2017): Brisbane 28th November -1st December, 2017, the 29th Australian Conference. Brisbane, Queensland, Australia. New York City: The Association for Computing Machinery (ICPS), S. 371-375.

Mirsch, Tobias / Lehrer, Christiane / Jung, Reinhard (2018): Making digital nudging applicable: The digital nudge design method. In: ICIS 2018 Proceedings. 
Rösler, Winfried (1983): Alltagsstrukturen-kognitive Strukturen-Lehrstoffstrukturen. Zur phänomenologischen Kritik an der kognitivistischen Lerntheorie. In: Zeitschrift für Pädagogik 29 (6), S. 947-960.

Sandfuchs, Barbara (2015): Privatheit wider Willen? Verbinderung informationeller Preisgabe im Internet nach deutschem und US-amerikanischem Verfassungsrecht. Dissertation. Tübingen: Mohr Siebeck.

Sandfuchs, Barbara / Kapsner, Andreas (2018): Privacy nudges: Conceptual and constitutional problems. In: Burk, Steffen / Hennig, Martin / Heurich, Benjamin / Klepikova, Tatiana / Piesga, Miriam / Sixt, Manuela / Trost, Kai Erik (Hg.): Privatheit in der digitalen Gesellschaft. Berlin: Duncker \& Humblot (Internetrecht und Digitale Gesellschaft, Band 10), S. 320-339.

Schöbel, Sofia / Barev, Torben / Janson, Andreas / Hupfeld, Felix / Leimeister, Jan Marco (2020a): Understanding user preferences of digital privacy nudges - A bestworst scaling approach. In: HICSS 2020 Proceedings, S. 3918-3927.

Schöbel, Sofia / Janson, Andreas / Jahn, Katharina / Kordyaka, Bastian / Turetken, Ozgur / Djafarova, Naza et al. (2020b): A research agenda for the why, what, and how of gamification designs results on an ECIS 2019 panel. In: CAIS 46. doi:10.17705/1CAIS. 04630.

Schomberg, Sabrina / Barev, Torben Jan / Janson, Andreas / Hupfeld, Felix (2019): Ansatz zur Umsetzung von Datenschutz nach der DSGVO im Arbeitsumfeld. Datenschutz durch Nudging. In: Datenschutz und Datensicherheit 43 (12), S. 774-780. doi:10.1007/s11623-019-1204-5.

Schwabe, Jürgen (1998): Der Schutz des Menschen vor sich selbst. In: JuristenZeitung, S. 66-75.

Skinner, Burrhus Frederic (1954): The science of learning and the art of teaching. In: Harvard Educational Review 24, S. 86-9.

Smith, H. Jeff / Dinev, Tamara / Xu, Heng (2011): Information privacy research. An interdisciplinary review. In: MIS Quarterly 35 (4), S. 989-1015.

Thaler, Richard H. / Sunstein, Cass R. (2008): Nudge. Improving decisions about bealth, wealth, and happiness. New Haven, Conn.: Yale Univ. Press. Online verfügbar unter http://www.loc.gov/catdir/enhancements/fy0833/2007047528-b. html (Abfrage am: 6.10.2020).

Thiel de Gafenco, Marian / Janson, Andreas / Schneider, Tim (2018): KoLeArn Smarte und kontextsensitive Aus- und Weiterbildung für die chinesische Industrie. In: DeLFI 2018 Proceedings.

Turland, James / Coventry, Lynne / Jeske, Debora / Briggs, Pam / van Moorsel, Aad (2015): Nudging towards security. Developing an application for wireless network selection for android phones. In: Proceedings of the 2015 British HCI Conference. New York, NY, USA: Association for Computing Machinery (British HCI '15), S. 193-201. doi:10.1145/2783446.2783588.

Tversky, Amos / Kahneman, Daniel (1974): Judgment under uncertainty. Heuristics and biases. In: Science (New York, N.Y.) 185 (4157), S. 1124-1131. 
Wang, Yang / Leon, Pedro Giovanni / Acquisti, Alessandro / Cranor, Lorrie Faith / Forget, Alain / Sadeh, Norman (2014): A field trial of privacy nudges for facebook. In: Jones, Matt / Palanque, Philippe / Schmidt, Albrecht / Grossman, Tovi (Hg.): CHI 2014, one of a CHInd. Conference proceedings : Toronto, Canada, April 26 - May 1, 2014 ; the 32nd Annual ACM Conference on Human Factors in Computing Systems. Association for Computing Machinery. New York, NY: Assoc. for Computing Machinery, S. 2367-2376.

Weinmann, Markus / Schneider, Christoph / vom Brocke, Jan (2016): Digital nudging. In: Business \& Information Systems Engineering 58 (6), S. 433-436. doi:10.1007/s12599-016-0453-1.

Zhang, Bo / Xu, Heng (2016): Privacy nudges for mobile applications. Effects on the creepiness emotion and privacy attitudes. In: Proceedings of the 19th ACM conference on computer-supported cooperative work \& social computing, S. 16761690.

Zuboff, Shoshana (2019): The age of surveillance capitalism. The fight for the future at the new frontier of power. London: Profile Books. 
